ISSN 1984-3755

Licenciado sob uma Licença Creative Commons

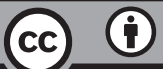

\title{
La ricezione del Concilio: mentalità, soggetti e tempo di un percorso laborioso ${ }^{1}$
}

A recepção do Concílio: mentalidade, temas e demorado caminho

\section{Gilles Routhier}

Doutor em Teologia pelo Instituto Católico de Paris e em História das Religiões e Antropologia Religiosa, pela Universidade de Paris - Sorbonne, professor no Instituto Católico de Paris e na Univeridade Laval, Québec, QC - Canadá, e-mail: gilles.routhier@ftsr.ulaval.ca

\section{Riassunto}

Questo documento affronta la complessa problematica della ricezione selettiva del Vaticano II Chiesa, focalizzando l'attenzione sul messaggio ricevuto e compreso ospitare uno stile nuova proposta della Chiesa in rapporto al mondo, valutando, quindi, l'incidenza del fattore nei processi di generazione. Solo la seconda e l'approfondimento di tale interesse rivela poco esplorato per rintracciare i suggerimenti più fruttuosi, invitando a considerare come difetti certamente positiva accoglienza della prima generazione post-conciliari hanno determinato l'atteggiamento decisamente più sospetti della seconda generazione. Questo conflitto suggerisce un cambiamento generazionale di

1 ROUTHIER, G. La ricezione del Concilio. Mentalità, soggetti e tempo di un percorso laborioso. Rivista del Clero Italiano, v. 91, n. 4, p. 263-285, 2010. Conferência pronunciada na jornada de estudo "0 que aconteceu no Vaticano II", que teve lugar na Universidade Católica de Milão em 2010. Texto gentilmente cedido à Pistis \& Práxis. 
approccio di combinare corpus: terza ed ultima fase di ricostruzioni storiche, forse è il momento di promuovere un incontro fecondo tra l'opera e il lettore, esplorando i temi del rapporto con la cultura, con il mondo e con il altri, in particolare vivono nei testi conciliari, e anche con altre, sensibili, cruciale anche per le nuove generazioni di giovani cristiani, uomini e donne.

Parole-chiave: Concilio. Front Desk. Generazione.

\section{Resumo}

O texto aborda seletivamente a complexa problemática da recepção eclesial do Vaticano II, focalizando a atenção na acolhida da mensagem conciliar entendida como proposta de um novo estilo eclesial na relação com o mundo, avaliando, portanto, a incidência do fator geracional nos processos de apropriação. Justamente o aprofundamento deste segundo e pouco explorado interesse revela-se a pista mais fecunda de sugestões, convidando a considerar como os defeitos da certamente positiva recepção da primeira geração pós-conciliar tenham determinado a atitude decididamente mais suspeitosa da segunda geração. Este conflito geracional sugere uma mudança de aproximação ao corpus conciliar: terminada a fase das reconstruções históricas, talvez tenha chegado o momento de favorecer um encontro frutuoso entre a obra e o leitor, explorando as temáticas da relação com a cultura, com o mundo e com os outros, particularmente vivas nos textos conciliares e, ainda que com outra sensibilidade, cruciais também para as novas gerações de jovens cristãos e cristãs.

Palavras-chave: Concílio. Recepção. Geração. Horizonte.

Non è facile affrontare in termini globali la ricezione del Vaticano II a motivo della smisurata ampiezza della questione. Ciò dipende in primo luogo dal fatto che bisogna chiedersi anzitutto quale sia l'oggetto offerto alla ricezione delle Chiese: se questo o quest'altro insegnamento del Vaticano II (e sono così numerosi e non vengono recepiti con le stesse modalità né allo stesso ritmo, a seconda dei differenti spazi ecclesiali); oppure il Vaticano II come evento che propone alla Chiesa un modo particolare, sinodale o 
conciliare, di trattare le questioni che la Chiesa cattolica deve affrontare; o ancora il Vaticano II come stile, cioè la forma particolare di discorso adottato dal Vaticano II. Certo, questi tre possibili oggetti non sono completamente isolabili gli uni dagli altri, ma la porta d'ingresso che si sceglie determinerà in parte l'orientamento della ricerca e i suoi risultati. Inoltre, quale che sia l'oggetto preso in considerazione, il Concilio, il suo stile e i suoi insegnamenti sono oggi oggetto di aspri dibattiti ermeneutici.

La difficoltà di trattare la ricezione del Vaticano II dipende anche dai molteplici livelli a partire dai quali la si vuole considerare. È possibile infatti privilegiare la ricezione kerigmatica, cioè l'insieme degli sforzi dei pastori per promuovere il Concilio, che ha una dimensione istituzionale evidente, ma che ha anche la funzione di promuovere un nuovo stato d'animo, una nuova forma mentis. Oppure rivolgere l'attenzione alla ricezione sviluppata negli ambienti in cui si ritrovano quei mediatori culturali che sono i teologi e gli altri operatori della comunicazione che permettono la diffusione delle idee conciliari e formano i 'quadri intermedi'. Infine ci si può interessare a come l'insieme del popolo di Dio si appropria degli insegnamenti e dello stile conciliare, traducendolo nell'evoluzione delle pratiche, delle forme di vita, delle mentalità e delle spiritualità. Questi livelli di indagine non appartengono naturalmente allo stesso ordine e bisogna constatare che gli studi attuali hanno toccato soprattutto le prime due forme di ricezione perché è a tali livelli che è meglio documentata e ha lasciato più tracce, anche se è proprio l'ultimo livello - la ricezione pratica e la piena ricezione - che alla fine risulta più determinante.

La difficoltà dipende anche dallo spazio umano e culturale della ricezione. Come si è spesso osservato, il Vaticano II è il primo concilio della 'Chiesa-mondo' e la sua ricezione non evolve allo stesso ritmo e secondo le stesse modalità in Asia, in Africa, nello spazio Nord-Atlantico o in sud America, per limitarsi a questi grandi spazi culturali, che del resto non sono perfettamente omogenei. Inoltre, gli insegnamenti particolari del Vaticano II, assimilati in questi spazi rispettivi, non sono immediatamente gli stessi. Come vediamo, parlare della ricezione del Vaticano II in modo un po' preciso e in tutte le sue sfumature richiede che si indichi chiaramente di che cosa e a partire da dove si parla. 
Dato che la ricezione di un concilio corrisponde a come un concilio (insegnamenti, eventi e stile) si inscrive non solo nello spazio, ma anche nel tempo, pensare la ricezione significa quindi pensare l'evento nel chronos e il suo effetto nella durata. Ciò impone dunque di periodizzare il corso della ricezione e di osservarne le tappe (ROUTHIER, 2004, p. 225-243). Ci sono diversi modi di calcolare il tempo e di misurare la durata. Questi sono in buona parte legati all'oggetto che ci interessa o ai diversi livelli e ai diversi spazi considerati. Così, se si studia la ricezione dell'insegnamento del Vaticano II sul diaconato permanente, la periodizzazione sarà imposta dalla pubblicazione dei documenti che vi si riferiscono o dalle decisioni che lo riguardano e che scandiscono l'evoluzione di questa istituzione. Ugualmente, se si opta per lo studio della ricezione kerigmatica del Concilio, si periodizzerà in funzione degli attori della ricezione kerigmatica, essendo i pontificati o gli episcopati a determinare spesso la periodizzazione. Ciò detto, gli studi sulla ricezione dei concili hanno messo in primo piano altri due punti di riferimento che possono guidare nello stabilire una periodizzazione: il passaggio dei testi conciliari al diritto e la scomparsa della generazione che ha fatto il Concilio, e questo significa il passaggio a un'altra generazione.

Sembra in effetti che si sia superata una tappa nella ricezione di un concilio quando si passa dall'insegnamento del concilio alle figure istituzionali e al corpo giuridico che ne derivano. Le proposizioni conciliari sono allora espresse in forme istituzionali e in un linguaggio giuridico che ne determinano in larga misura la ricezione, in quanto le figure istituzionali (ivi compresi i rituali) e il corpus giuridico rappresentano mediazioni determinanti tra l'insegnamento di un concilio e le pratiche effettive. È questo il caso, per esempio, quando si passa dal discorso conciliare sulla partecipazione attiva nella liturgia ai rituali e alla pubblicazione dell'insieme delle norme liturgiche. Le forme istituzionali, che costituiscono quadri d'azione e forme nelle quali si calano le pratiche, contribuiscono potentemente a plasmare le mentalità.

In termini generali, possiamo dire che questa tappa è alle nostre spalle e si è conclusa con la pubblicazione del Codice di diritto canonico, che assume le norme emanate dopo il Concilio nei diversi ambiti della vita ecclesiale (formazione dei sacerdoti o dei diaconi, in materia liturgica, 
sull'ecumenismo, la conduzione dei sinodi, ecc.), e la pubblicazione dei diversi rituali. Certo, tutto ciò non ha smesso di cambiare (ne offre un esempio il motu proprio Summorum pontificum o la revisione recente della ratio studiorum per la formazione dei sacerdoti), ma il periodo segnato da una grande produzione legislativa (direttori, norme, istruzioni) o dalla creazione di nuove figure istituzionali e dalla produzione di libri liturgici è ormai alle nostre spalle. Da questo punto di vista possiamo dire che siamo entrati in una nuova fase della ricezione.

Si afferma anche che una tappa è superata quando la generazione di coloro che hanno fatto un concilio si spegne e lascia il posto a un'altra generazione che non è stata impegnata nel dibattito conciliare e che non è per ciò segnata allo stesso modo da quell'evento. I membri di questa generazione sono meno portati, si dice, a prolungare extra aulam i dibattiti che hanno occupato il periodo conciliare. La nuova generazione è portatrice di un'altra esperienza, di un'altra sensibilità spirituale ed ecclesiale, appartiene a una diversa cultura plasmata da un altro contesto. È quanto accade oggi, quando tutti i protagonisti sono usciti di scena, benché Benedetto XVI abbia partecipato al Concilio, non però a titolo di padre conciliare.

Comunque stiano le cose sulla questione dell'appartenenza di Benedetto XVI alla generazione che ha fatto il Concilio, è indubbio che il Vaticano II sia oggi affidato alle mani di un'altra generazione e non è facile per gli esponenti della nuova generazione riappropriarsi, assimilare, far proprio, insomma recepire il Vaticano II col quale intrattengono un rapporto distante e di cui spesso conoscono ben poco l'insegnamento e la storia. Esso non è tuttavia ridotto allo statuto di una realtà astratta da cui questa generazione sarebbe completamente separata. In effetti, per un certo numero di essi, il Vaticano II si identifica con l'epoca dei genitori - che spesso non hanno trasmesso loro la fede - dalla quale vogliono prendere le distanze e con la quale spesso vogliono rompere. Per i giovani occidentali, il Concilio appartiene al mondo dei baby-boomers che sono usciti dalla religione emancipandosi dalla religione dei padri. Ai loro occhi, i baby-boomers non lasciano loro alcuna eredità cristiana. Così, se si sono convertiti in età giovanile, riscoprendo il cristianesimo e la Chiesa, sono tentati di assumere una posizione contraria rispetto a quella dei genitori. 
Il periodo conciliare è allora sentito come responsabile del vuoto spirituale che provano e della disfatta della Chiesa.

La mia frequentazione dei giovani all'università o altrove mi ricorda continuamente l'urgenza di pensare la ricezione del Vaticano II da parte di una nuova generazione e mi induce a riflettervi, lavoro che ho cominciato in una conferenza tenuto alla Cattedra Tillard l'anno scorso (ROUTHIER, 2009, p. 237-255), riflessione che è urgente proseguire poiché la questione mi sembra rappresentare oggi, e di gran lunga, la più importante sfida al capitolo della ricezione del Vaticano II. Diverse altre questioni, se si vuole assicurare la perennità del Concilio nel corpo ecclesiale, hanno anch'esse un certo carattere di gravità e di urgenza ed enumerarle porterebbe alla stesura di un lungo elenco. Qui mi soffermerò su quegli attori particolari della ricezione del Vaticano II che sono i giovani cattolici attivi e militanti dei paesi occidentali, e su di un momento particolare della ricezione del Vaticano II, il periodo contemporaneo. Così facendo, mi allontano dagli studi sulla ricezione che si interessano ai dibattiti tra teologi o storici o ai mutamenti che dipendono da parole e gesti degli personaggi in carica nei diversi organismi del governo della Chiesa cattolica, in particolare del suo governo centrale. Questa presa di distanza non significa comunque che non vi siano legami tra gli atteggiamenti e le reazioni caratteristiche della giovane generazione di cattolici e quelli che ritroviamo in certi responsabili ecclesiali. La scelta di un periodo determinato mi dispensa anche dal tracciare di nuovo l'insieme del percorso della ricezione del Vaticano II e dall'entrare nel dibattito sulla periodizzazione di quel complesso percorso. Infine, la scelta mi porterà a fissarmi su due elementi centrali e caratteristici del Vaticano II (insegnamento, evento e stile) offerti alla ricezione: il rapporto con gli altri e con il mondo e la forma di espressione del cristianesimo che ne risulta.

Ho così delimitato il quadro di questo studio precisando anzitutto il guadagno offerto dal Vaticano II sul quale si concentrerà l'attenzione che nel nostro caso non è un insegnamento particolare, ma la proposta di un nuovo rapporto con il mondo e con gli altri, che si esprime attraverso tutto il Concilio e che induce un nuovo 'stile' di discorso -, quindi i soggetti della ricezione - i giovani cattolici occidentali militanti -, e infine il periodo. 


\section{Una generazione che costruisce la sua identità cattolica in un nuovo rapporto con il mondo e con gli altri}

I tre elementi citati interagiscono tra loro come parti di uno stesso sistema. In effetti, ciò che definisce la generazione di giovani cattolici militanti è il fatto di vivere in un ambiente che, benché profondamente segnato dal cristianesimo, non è più 'ufficialmente' cattolico e di dover costruire la loro identità cattolica (ed esprimerla) in un rapporto con gli altri che è profondamente cambiato dopo il Concilio Vaticano II. È questa esperienza a segnarli, esperienza che dobbiamo anzitutto approfondire, prima di andare oltre, perché ciò che costituisce una generazione è appunto un'esperienza condivisa.

Uno dei primi ricercatori ad aver studiato il fenomeno delle generazioni, José Ortega y Gasset, ritiene che il fattore generazionale sia non solo il più importante sul piano dell'evoluzione della storia, ma che ogni generazione rappresenti un modo particolare di sentire e di sperimentare il mondo e le cose (ORTEGA Y GASSET, 1994). Proseguendo questi studi, Karl Mannheim distingue chiaramente le "generazioni" dai "gruppi sociali" (MANNHEIM, 2008) e osserva che il fatto di avere la stessa età e di essere nati nella stessa epoca non basta a fare una generazione. Nello stesso senso, O. Galland distingue la nozione di "coorte" da quella di "generazioni” (GALLAND, 2001, p. 107-108)2. In effetti bisogna distinguere una generazione dall'effetto di età ${ }^{3}$ in quanto il fatto che le persone siano nate negli stessi anni, invecchino insieme e scompaiano nella stessa epoca è insufficiente perché si possa parlare di una generazione. Perché si abbia una "generazione effettiva", occorrono dei legami tra le persone e i legami risultano dal fatto che tali persone sono in condizione di sperimentare gli

2 L'autore distingue anche tra le generazioni storiche, le generazioni "genealogiche" (persone che, avendo vissuto la stessa esperienza, ne hanno conservato un senso di identità molto forte in quanto si definiscono in riferimento a tale esperienza che attiene ai rapporti tra genitori e figli) e le generazioni sociologiche (persone nate nella stessa epoca e che hanno vissuto le stesse esperienze, senza che questo nutra la coscienza di appartenenza a un gruppo o definisca in modo consapevole la loro identità).

3 Si veda in proposito KESSLER, D.; MASSON, A. Cycles de vie et générations. Paris: Economica, 1985; GALLAND, 0. Sociologie de la Jeunesse. Paris: Armand Colin, 1997. p. 117. in particolare il cap. III, Âge de la vie et génération. p. 103-132 e le sue distinzioni tra "effetti di età, effetti di coorte, effetti di periodo".

Rev. Pistis Prax., Teol. Pastor., Curitiba, v. 4, n. 2, p. 475-501, jul./dez. 2012 
stessi eventi e abbiano sviluppato una stessa coscienza storica. Bisogna inoltre precisare che le persone appartenenti a una stessa generazione, anche se sentono delle affinità, non trattano tutte le esperienze comuni nello stesso modo, anche se su diverse questioni di fondo vi è un comune modo di sentire e di comprendere le cose. È negli atteggiamenti che precedono le opzioni politiche o religiose che vanno cercati i tratti comuni a una generazione.

Con C. Attias-Donfut, intendiamo quindi per generazione "un insieme di persone all'incirca della stessa età ma per le quali il principale criterio di identificazione risiede in esperienze storiche comuni da cui hanno tratto una comune visione del mondo" (ATTIAS-DONFUT, 1991, p. 59) ${ }^{4}$. Ciò corrisponde alla posizione del sociologo Fernand Dumont per il quale "si appartiene a una generazione quando si è vissuta con altri una stessa fase storica, quando si condividono analoghi riferimenti di memoria" (DUMONT, 1986, p. 16) . I ricercatori concordano sul fatto che la coscienza generazionale si cristallizza nel corso dell'adolescenza e della giovinezza e tale coscienza dipende dal fatto di essere stati profondamente segnati da un'esperienza. Infine, ogni generazione ha coscienza della propria identità in quanto nutre il suo senso di discontinuità in rapporto al passato, e trova la propria definizione "non più nei lasciti delle generazioni precedenti” (GALLAND, 2001, p. 111), ma nella propria reazione alla situazione in cui è inserita.

In simili circostanze, le nuove generazioni provano quel senso di tabula rasa che le obbliga a inventare i propri quadri di riferimento, spesso in opposizione radicale con quelli che i vecchi proponevano loro [...]. L'esperienza socializzatrice è interrotta dalla perdita di legittimità della generazione più vecchia (GALLAND, 2001, p. 111-112).

Tutto ciò contribuisce a giustificare la scelta che facciamo di studiare in modo particolare la ricezione del Vaticano II da parte della nuova generazione. In effetti, una generazione si definisce all'epoca della

4 Della stessa Autrice si veda anche Sociologie des générations: l'empreinte du temps. Paris: PUF, 1988. p. 249 e la voce "Générations".

5 Altrove l'autore dirà: “Una generazione è composta di contemporanei che hanno vissuto simili eventi storici. Una generazione forma un raggruppamento sociale di una specie particolare", p. 20. 
giovinezza e la coscienza storica che la definisce risulta legata al processo di socializzazione, in quanto una nuova generazione non prova la realtà del mondo e la vita nello stesso modo della precedente. Anzi, spesso vi si oppone. È proprio questo il caso dei giovani cattolici convertiti.

Dobbiamo ora chiederci quale sia l'esperienza storica comune alla generazione di giovani cattolici occidentali militanti (non sono però certo che l'esperienza sia condivisa dai giovani italiani). Il tratto comune a questa generazione mi sembra essere di non avere ricevuto la fede e l'appartenenza alla Chiesa dal mondo nel quale sono stati calati e dalla cultura in cui sono stati immersi fin dall'infanzia e di provare un disagio in quel mondo che è loro dato in eredità. Così, il mondo che abitano e la cultura che ha pervaso i primi vent'anni della loro vita non appaiono loro fin da subito come gradevoli, perché non hanno dato loro la fede e non hanno permesso che facessero l'esperienza della vita in Chiesa, cose che oggi considerano beni preziosi. Inoltre, convertiti al cattolicesimo da giovani, si ritrovano generalmente in minoranza in un ambiente non cristiano e pluralista. È così che, in ragione della loro conversione, l'esperienza di socializzazione offerta dalla famiglia, dalla società e dalla cultura che avevano conosciuto durante l'infanzia è rotta, il che comporta una perdita di legittimità della generazione precedente e dei suoi riferimenti. Di conseguenza il lascito rappresentato dal Concilio Vaticano II - ormai identificato con la generazione che non si è preoccupata del loro diventare cristiani - è in pericolo. Insomma, questa esperienza li induce ad avere un'immagine del Concilio Vaticano II che li porta spontaneamente a non recepirlo, non giudicandolo opportuno per loro; la ricezione infatti comporta sempre un giudizio sull'opportunità del bene che ci viene offerto. In ragione del fattore generazionale, il Vaticano II giunge loro attraverso un'immagine che lo snatura, il che non manca di complicare le cose.

Inoltre il loro status di minoranza, caratterizzato da valori e comportamenti che non sono socialmente attestati, li porta ad assumere una posizione particolare nei confronti degli altri, della cultura circostante e del mondo che abitano, il cui asse dominante si orienta sul confronto e la polemica, atteggiamento rafforzato dal loro status di convertiti. L'opzione recente in favore del Cristo e del suo Vangelo e l'appartenenza spesso recente alla Chiesa ha per conseguenza che la loro identità cattolica spesso 
è insicura. Hanno inoltre più bisogno di posizioni nette, di convinzioni forti e di verità chiare. Sono continuamente rimessi in discussione dal loro ambiente, cosa che li costringe ad affermare in modo reciso la loro posizione e a manifestare, talora senza modestia, la loro identità.

Il loro rapporto con il mondo e con gli altri, elemento centrale del Vaticano II, è così molto diverso da quello sviluppato dalla generazione conciliare. In Occidente, chi ha vissuto all'epoca del concilio aveva ricevuto la fede dalla cultura e dal mondo in cui era immerso nell'infanzia. Inoltre apparteneva a un mondo cristiano dove la sua fede cristiana era socialmente attestata. E infine apparteneva alla maggioranza e si definiva più per imitazione che per distinzione o separazione. Quanto ai giovani convertiti, essi provano un senso di discontinuità in rapporto alla generazione che li precede, trovando ciò che li definisce, "non più nei lasciti delle generazioni precedenti" (GALLAND, 2001, p. 111), ma nella reazione alla situazione in cui sono collocati.

In circostanze del genere, le nuove generazioni provano quel senso di tabula rasa che le obbliga a inventare i propri quadri di riferimento, spesso in opposizione radicale con quelli che i vecchi proponevano loro [...]. L'esperienza socializzatrice è interrotta dalla perdita di legittimità della generazione più vecchia (GALLAND, 2001, p. 111-112).

L'esperienza storica dei giovani convertiti, quella di non condividere convinzioni comuni, di doversi aprire in solitaria un cammino in mezzo a una società pluralista dagli ideali incerti e di essere continuamente rinviati al dominio del mercato e del consumo, non è esclusiva dei convertiti. Gli studi attuali su quanto si esprime negli ambienti artistici indicano che l'esperienza di delusione nei confronti del mondo moderno e l'esperienza di vuoto esistenziale appartengono più ampiamente ai loro coetanei ${ }^{6}$. Di fatto è tutta una generazione a esprimere il suo male di vivere e l'insoddisfazione profonda nei confronti del mondo che le è stato

$6 \mathrm{Si}$ veda in particolare MATTE, I. Malaise existentiel et discours apocalyptique dans la jeune chanson québécoise. In: MAGER, R.; CANTIN, S. Modernité et religion au Québec. Où en sommes-nous? Québec: PUL, 2009. p. 165-179; MATTE, I. Rite de passage en contexte moderne: l'exemple de la scène musicale underground montréalaise. 2000. 130 f. Tesi (di Laurea Anthropologie) - Dipartimento di Antropologia, Università Laval, 2000. 
dato in eredità dai predecessori. Su questo fondo di coscienza storica comune, i giovani cristiani (convertiti, cattolici o appartenenti ad altri gruppi cristiani) danno una soluzione originale a questa difficoltà: il ritorno al Cristo permetterà una rigenerazione della società e della cultura.

L'esperienza storica comune alla nuova generazione di giovani cristiani che voltano le spalle al mondo che non ha dato loro una ragione di vivere, esperienza storica che mi sembra propriamente occidentale, ha importanti conseguenze sul loro modo particolare di entrare in rapporto con gli altri e con il loro ambiente culturale. Ha conseguenze importanti anche sulla costruzione della loro identità spirituale e religiosa e sul modo di esprimere il loro credere. Se vogliamo interpretare correttamente l'attuale ricezione del Concilio Vaticano II da parte di questa nuova generazione, dobbiamo innanzitutto riconoscere che, sul piano dell'orientamento spirituale, abbiamo propriamente a che fare con una nuova generazione e che, cosa ancor più determinante, a monte degli atteggiamenti e delle modalità di espressione religiosa, vi è una diversa esperienza della cultura, del mondo, della società, del pluralismo religioso e delle convinzioni.

Dobbiamo davvero constatare che l'attenzione rivolta al fattore generazionale è stata minore nella Chiesa $^{7}$ rispetto alla società, dove si è estremamente attenti ai cambiamenti generazionali, dove si analizza il modo di comprendere il mondo e di rapportarsi a esso della generazione $\mathrm{X}$ e della generazione $\mathrm{Y}^{8}$. Credo però che non si possa trascurare il concetto di generazione e un'approfondita esplorazione dell'esperienza di

7 In Quebec, si vedano gli studi del gruppo diretto da GRAND'MAISON, J. Le défi des générations. Enjeux sociaux et religieux du Québec d'aujourd'hui. Montréal: Fides, 1995. Si veda anche il contributo di PELLETIER, D. La crise catholique. Paris: Payot, 2002.

8 La definizione "generazione X" viene dalla classificazione di HOWE, N.; STRAUSS, W. Che hanno pubblicato una serie di volumi sulla questione delle generazioni negli Stati Uniti. Si tratterebbe della generazione tra i baby-boomers e la generazione Y (o "figli del millennio", "net generation", o "digital natives") composta di giovani nati a partire dagli anni 1980. Si HOWE, N.; STRAUSS, W. 13th gen: abort, retry, ignore, fail? New York: Broadway Books, 1993; HOWE, N.; STRAUSS, W. The fourth turning: an American prophecy. New York: Vintage Books, 1997; HOWE, N.; STRAUSS, W. Millennials rising: the next great generation. New York: Vintage Books, 2000; HOWE, N.; STRAUSS, W. Millennials go to college: strategies for a new generation on campus. Washington: American Association of Collegiate Registrars, 2003; HOWE, N.; STRAUSS, W. Millennials and the pop culture: strategies for a new generation of consumers. Great Falls: Lifecourse Associates, 2006a; HOWE, N.; STRAUSS, W. Millennials go to college surveys and analysis: from boomer to gen-x parents. Great Falls: Lifecourse Associates, 2006b; College Student And Parent Surveys, 2007.

Rev. Pistis Prax., Teol. Pastor., Curitiba, v. 4, n. 2, p. 475-501, jul./dez. 2012 
tale generazione se si vuole pensare con maggiore sottigliezza il periodo post-conciliare e la ricezione del Vaticano II oggi nei paesi più segnati dalla iper-modernità. In effetti, la ricezione del Vaticano II non si lascia ridurre al passaggio da una fase di espansione a una di contrazione, o da una fase di esaltazione a una di delusione, fasi che sarebbero seguite oggi da un periodo di sintesi come hanno proposto certe periodizzazioni (POTTMEYER, 1985, p. 43-65) ${ }^{9}$. La ricezione non è neppure semplicemente dipendente da una opposizione tra conciliari e anti-conciliari che prolungherebbe fino a oggi l'opposizione tra maggioranza e minoranza nel Concilio. Vi sono generazioni, vale a dire esperienze storiche, che determinano il nostro rapporto con il Concilio, esperienze che determinano anche il nostro rapporto con il mondo e la cultura circostante, esperienze che sono precedenti alla definizione del nostro rapporto col Concilio sulle questioni che qui esaminiamo: il rapporto con gli altri e col mondo e la forma di espressione del cattolicesimo. Certo, non bisogna ridurre tutto al fattore generazionale - vi sono veri e propri oppositori al Vaticano II la cui posizione anti-conciliare non va attribuita al gap generazionale -, ma di questo occorre tener conto.

\section{Una ricezione contrastata del Vaticano II da parte di generazioni molto diverse}

Così, se la si guarda a partire da questa prospettiva, si può dire che la ricezione del Vaticano II ha inizialmente beneficiato - e ancora beneficia nei momenti di crisi - di un forte sostegno della generazione dei baby-boomers anche se essi hanno vissuto dopo il Concilio un periodo di disillusione - ma non di indifferenza - in rapporto ai cambiamenti della Chiesa cattolica, il che corrisponde forse alla fase di delusione di cui parla Pottmeyer. In seguito, le intuizioni conciliari hanno avuto molte difficoltà a incarnarsi in forme di vita e modalità di espressione della fede in ragione del sentire anti-istituzionale e anti-tradizionale dominante nel corso

9 Si veda POTTMEYER, H. J. Vers une nouvelle phase de réception de Vatican II. Vingt ans d'herméneutique du Concile. In: ALBERIGO, G.; JOSSUA J.-P. La réception de Vatican II. Paris: Cerf, 1985. p. 43-64. 
degli ultimi anni Settanta. In parte, la ricezione del Vaticano II si è giocata qui poiché il Concilio (o la sua efficacia) richiedeva la istituzionalizzazione dei fermenti conciliari in forme di vita rinnovate e in nuovi modi di espressione della fede, al di là anche di quelli che erano proposti dal rinnovamento delle figure istituzionali previste e pensate tra i responsabili del governo della Chiesa cattolica. Qui è interessante non solo occuparsi della ricezione kerigmatica operata dai pastori - l'attuazione del Vaticano II - ma esaminare come i fedeli abbiano fatto propri i fermenti spirituali offerti dal Concilio.

Per la generazione alla quale il Vaticano II è stato inizialmente offerto, la spontaneità, più della ripetizione, era virtù. La libertà, più della tradizione, era la regola. L'autenticità, più dei codici, doveva guidare le pratiche. Lo spirito, più della lettera, guidare i comportamenti. L'immediatezza dei rapporti sociali, più delle regole istituzionali, doveva ispirare i comportamenti. Il gesto vero, più del ripetere le pratiche, diveniva segno di un agire vero. Ciò che si sentiva e provava, più di ciò che era definito, sembrava suonare giusto. L'esperienza, più della norma, sembrava esprimere il vero. La creatività e la libera espressione erano chiamate $\mathrm{a}$ subentrare alle forme imposte e ai quadri definiti. La sincerità prevaleva sulle norme. L'interiorità più dell'esteriorità era incoraggiata, la giustizia e la carità più delle pratiche cultuali e ascetiche riconosciute e accreditate. Quella generazione, che si è distaccata dalle istituzioni - o che le ha tenute a distanza - e affrancata dalle pratiche formalizzate e ripetitive, ha conservato nella memoria il Vangelo portato nella Chiesa cattolica come un riferimento importante, ma il riferimento non si è tradotto in pratiche regolari e appartenenze forti.

Insomma, si osserva una sconnessione tra il programma di riforme istituzionali di grande portata condotto soprattutto a partire dal governo centrale della Chiesa e il sentire anti-istituzionale che costituisce un tratto culturale di quel periodo. Si offrono da un lato forme rinnovate mentre dall'altro si vogliono inventare o creare nuove forme. È la mancata coincidenza o assenza di sincronia tra la ricezione kerigmatica e l'ethos spirituale e culturale (o l'orizzonte d'attesa) della prima generazione che ha recepito il Vaticano II a porre un problema particolare. Non è tuttavia eccezionale che vi siano sensibilità diverse tra i pastori incaricati di 
promuovere le decisioni di un concilio e il popolo cristiano, o addirittura tra diversi gruppi responsabili della attuazione delle decisioni conciliari. Ricordiamo che dopo il concilio di Trento, come gli storici hanno mostrato, vi furono diverse transazioni tra gli sforzi riformatori profusi dai pastori al seguito del Concilio e le aspirazioni spirituali latenti dell'epoca. In effetti, gli storici hanno mostrato che il clero post-tridentino aveva dovuto fare numerosi compromessi e acconsentire a vari accomodamenti nell'attuazione della Riforma tridentina: approvazione del culto dei santi, della credenza nei miracoli, delle domande di beni materiali, che pure erano tutte realtà estranee a quella Riforma. Per designare l'adattamento del clero alla religione dei fedeli, Jean Delumeau ha parlato di una volontà di acculturazione da parte del clero (DELUMEAU, 1975, p. 9). Mediante un simile adattamento, il popolo cristiano aveva potuto, in cambio, assimilare le nuove devozioni e le relative pratiche, proposte dai movimenti della Controriforma, divenute comuni nel XVIII secolo. Le transazioni tra i pastori e il popolo dei fedeli sono dunque numerose e non avvengono a senso unico. Jean Delumeau osservava, per esempio, in Bretagna, "una sorta di accomodamento per distorsione del cristianesimo e delle mentalità popolari" (DELUMEAU, 1975, p. 12). Alain Lottin arrivava a conclusioni analoghe, notando che in Fiandra e nell'Ainaut si può constatare un incontro tra la religione popolare e la Controriforma, incontro che si esprime nelle modalità dell'adattamento, dell'impregnazione dell'una da parte dell'altra e di modificazione progressiva (LOTTIN, 1979, p. 58). Alcuni vedono, nei cambiamenti conseguenti i movimenti di riforma, che segnano il cattolicesimo e il protestantesimo e che si estendono su di un lungo periodo che va dal XV al XVII secolo, sia epurazioni riuscite degli elementi popolari dal cristianesimo, sia una repressione di tali elementi.

Quanto possiamo osservare per il momento in seguito al Vaticano II - ma è ancora presto per trarre conclusioni - è che ancora non si assiste a un incontro riuscito tra le riforme proposte - soprattutto a partire dagli organismi centrali del governo della Chiesa cattolica - e le mentalità popolari. Non possiamo trarre subito la conclusione di una non-ricezione del Vaticano II. Quel che non coincide in effetti sono le riforme istituzionali che ne sono state tratte e l'appropriazione che i fedeli hanno fatto del Vaticano II in un contesto culturale particolare. Non c'è da stupirsi di 
questa non-coincidenza, ma c'è da augurarsi che delle transazioni possano permettere scambi tra le due cose.

$\grave{E}$ in questo contesto di 'appuntamento mancato' che il movimento di applicazione del Concilio, realizzato soprattutto a partire dagli organismi centrali del governo della Chiesa cattolica, ha funzionato. L'applicazione è consistita nell'instaurare le figure istituzionali e le forme liturgiche previste dal Vaticano II. La fase di applicazione ha prodotto un numero impressionante di riforme istituzionali che non sempre ha toccato i fedeli che non aspiravano a vivere il cristianesimo inscrivendo il loro agire nelle istituzioni e in forme spirituali e di pietà predeterminate, sia pure rinnovate. Così, la risposta alle riforme non è stata quella che ci si aspettava, il che ha presto indotto alcuni attori a pensare che non occorresse continuare sulla via delle riforme, non essendo i risultati all'altezza delle promesse. Abbastanza presto si sono levate voci che lasciavano intendere che si era sbagliata direzione e che bisognava tornare indietro.

In effetti, la sfasatura tra le riforme proposte e la loro appropriazione ha portato in molti casi a una ritirata, dato che le proposte di partenza conoscevano in gran parte mutamenti che ne riducevano l'apertura e le possibilità. In cambio, la creazione sul piano locale di forme di vita, proprie tradizioni e pratiche a partire dall'insegnamento del Vaticano II e dalle sue intuizioni spirituali ha avuto generalmente minore riuscita. Certo, vi sono state varie iniziative interessanti e un buon numero di esperienze di qualità, che però non si sono inscritte nella durata istituzionalizzandosi. Inoltre si è ristretto sempre più lo spazio di sperimentazione e di creazione che era stato riconosciuto alle Chiese locali, per il venir meno dell'incoraggiamento alla vita sinodale sul piano locale, alla sperimentazione liturgica, ecc.

Il delicato passaggio dall'evento all'istituzione durante la prima fase della ricezione del Concilio è risultato complicato da diversi fattori. L'uscita dalle forme antiche - identificate nel clericalismo, nel trionfalismo e nella giuridicità denunciati in Concilio - non ha portato da subito a vivere nelle forme nuove proposte dal governo centrale della Chiesa cattolica o, più di rado, sorte dai diversi ambienti sul piano locale. È l'istituzionalizzazione di una nuova figura del cattolicesimo ad aver costituito un problema ed è il problema dell'istituzionalizzazione della vita cristiana e della fede a venire di nuovo posto con la nuova generazione. 
In effetti, i membri della nuova generazione non hanno trovato nella loro famiglia o nel loro universo sociale pratiche istituite e regolari (pratiche di pietà, pratiche ascetiche, ecc.), comportamenti definiti e stabiliti, forme di vita stabili e quadri di pensiero che fissino in modo preciso la vita cristiana. Insomma, non hanno potuto inscrivere gli elementi della vita cristiana che è stata loro trasmessa in una tradizione. Scoprendo o riscoprendo il cattolicesimo durante la giovinezza in occasione di un'esperienza forte, vero e proprio momento di conversione che li pone sulla strada del ritorno, talvolta del loro battesimo o del loro ingresso in seminario o nella vita religiosa (comunità nuove o di lunga tradizione), il cattolicesimo di riferimento dei genitori appare loro vago e poco consistente. Non possono più viverne e si mettono alla ricerca di forme istituzionali ben stabilite, visibili e autorizzate, di pratiche stabili e codificate, di punti di riferimento chiari, dalle linee nitide e ben definite. La ricerca di un'identità cattolica chiaramente definita è il risultato della loro esperienza in una società tollerante, che ha fatto loro vivere una erranza spirituale e un'incertezza sul piano delle convinzioni, una società pluralista dove sono posti di fronte, nella loro ricerca identitaria, a tradizioni religiose che non mancano né di visibilità né di certezza, che non hanno timore di affermarsi in modo a volte clamoroso, e si presentano con una sicurezza a tutta prova.

\section{Interazioni complesse}

Non bisogna tuttavia limitare la nostra analisi alla messa in scena di queste due generazioni dalle esperienze contrastanti. In effetti d'altronde, nel corso dello stesso periodo, ai margini, era in atto un lavoro di istituzionalizzazione del cattolicesimo. Esso conosce almeno due versanti: (1) una istituzionalizzazione che assume la forma di una reazione alle nuove forme proposte (al piano liturgico, a quello catechetico e a quello del governo ecclesiale) e di un rifiuto della nuova figura del cattolicesimo che tenta di disegnarsi. Si tratta in questo caso di una istituzionalizzazione per restaurazione e regressione, per rigetto e di rifiuto. (2) Si osserva anche un'istituzionalizzazione per invenzione carismatica e bricolage. La si trova 
soprattutto nei movimenti e nelle comunità nuove - difficili da trattare in modo globale - che presto si affermeranno come un vettore importante della ricezione del Vaticano II. Queste comunità sorgeranno intorno a figure carismatiche portatrici di una vera e propria intuizione spirituale. Si tratta di fenomeni essenzialmente urbani e poco legati al territorio, come sono invece le diocesi e le parrocchie. Le forme di vita elaborate in questi gruppi si ispirano a diverse epoche e a varie tradizioni e riuniscono in un nuova combinazione elementi apparentemente eterocliti, alla maniera delle costruzioni post-moderne.

Sono questo contesto e questa esperienza generazionale insieme a determinare un nuovo momento nella ricezione del Vaticano II, in particolare relativamente alla questione del rapporto col mondo, con la cultura e con gli altri. Tuttavia, prima di arrivarci più direttamente, bisogna aggiungere altri elementi. In effetti, la novità della situazione non concerne solo i cambiamenti contestuali che ho qui sommariamente presentato, non più semplicemente la variabile generazionale che ho messo in evidenza. Vi sono inoltre transazioni complesse che producono anch'esse la novità della situazione. In effetti, la nuova generazione intrattiene rapporti con altri gruppi di attori diversamente collocati nello spazio ecclesiale. Vi sono da una parte i gruppi tradizionalisti di tendenza fondamentalista e integralista, e dall'altra attori di tendenza sostanzialmente conciliare ma inquieti per i mutamenti recenti della cultura occidentale e della Chiesa cattolica. Bisogna aggiungere inoltre la tendenza, che è propria alle istituzioni, a volersi perpetuare e a resistere al cambiamento. Così, come osserva John O’Malley per il periodo che segue il concilio di Trento, bisogna fare i conti anche, dopo il Vaticano II, con la resilienza di un'istituzione e del suo stile ${ }^{10}$.

Insomma, questa generazione è anche prodotta da altre forze e, in una certa misura, è in balìa di queste altre forze e viene strumentalizzata dai gruppi che vogliono approfittare della sua ricerca di sicurezza e di certezza. Così, l'opposizione radicale al Concilio esiste fin dagli inizi e

10 Si veda O'MALLEY, J. The resiliency of the institution and its style. In: O'MALLEY, J. Four cultures of the west. London: Harvard University Press, 2004. p. 115-125.; O'MALLEY, J. Quattro culture dell'Occidente. Milano: Vita e Pensiero, 2007. 
si esprime in particolare attraverso la Fraternità san Pietro e alcuni altri gruppuscoli ${ }^{11}$. È marginale e, benché rumorosa, non rappresenta una for$z a$ in grado di influenzare il corso delle cose e di porsi al centro del gioco "politico" durante il periodo della ricezione del Concilio fintanto che, e per tutto il tempo che, non arriva ad allearsi con altri gruppi e a beneficiare del loro sostegno. L'alleanza non è stata possibile durante la prima fase della ricezione, ma oggi constatiamo che il rapporto di forze è cambiato. I gruppi di questa tendenza si oppongono irriducibilmente al mondo moderno che condannano senza sfumature e con molta violenza.

A ciò si aggiunge un movimento di resistenza più sordo al Concilio, movimento paragonabile a quello che si trova in altre epoche in seguito a un concilio. Si tratta semplicemente, nel nostro caso, di una resistenza al cambiamento che esprime la difficoltà inerente a tutte le istituzioni a cambiare le vecchie abitudini e ad adottare una nuova forma mentis. Questo movimento di inerzia ha portato a una ricezione minima di certe disposizioni del Concilio e ha potuto incoraggiare certi movimenti di opposizione. Tale resistenza 'burocratica' si è anche adoperata a limitare le iniziative o le innovazioni provenienti dalla 'periferia'. E in più la ricerca di certezza e di sicurezza spalanca la strada a quanti vogliono affermare la propria autorità più che educare alla libertà.

L'elemento più importante, senza dubbio, sono stati i cattolici 'inquieti' del divenire del cattolicesimo e della società moderna e che si sono adoperati a riorientare la ricezione del Concilio. Le iniziative più importanti in tal senso sono quelle di Giovanni Paolo II. Questo Papa si è sforzato di ridare maggiore dinamismo e maggiore visibilità al cattolicesimo. Ha favorito l'emergere di un cattolicesimo affrancato dai suoi complessi, che manifesta un'identità chiara, che si esprime con forza attraverso un discorso e delle pratiche di massa. Questo programma fa leva su diverse forze vive, soprattutto le comunità nuove e i giovani. Nel momento in cui la Chiesa cattolica sembrava mancare di coerenza e di chiara identità,

${ }^{11}$ Si veda BUONASORTE, N. Tra Roma e Lefebvre: II tradizionalismo cattolico italiano e il Concilio Vaticano II. Roma: Studium, 2003. Si vedrà in particolare il cap.III le cui prospettive vanno oltre l'Italia. Per il Brasile, si vedrà la conclusione della recente tesi di Rodrigo Coppe Caldeira, Os Baluartes da Tradição: a antimodernidade católica brasileira no Concilio Vaticano II, nel Programma di Post-Graduazione di Scienze della Religione dell'Università Federale di Juiz Juiz de Fora. 
ha invitato i cattolici a serrare i ranghi intorno a posizioni nette che in certi momenti non mancavano di prendere di petto i valori e i comportamenti attestati nella cultura occidentale. Ciò detto, nessuno può mettere in discussione la fedeltà di Giovanni Paolo II al Concilio che egli presenta come una bussola sicura per guidare la Chiesa nel terzo millennio. Ne ha fatto il fondamento del programma del suo pontificato. Ancor più, se Giovanni Paolo II non teme di polemizzare con la cultura occidentale, non lo si potrà mai sospettare di vivere nella paura dell'altro e di avere difficoltà a incontrarlo sul suo terreno. Ugualmente esemplare è il suo impegno a incontrare i credenti di altre religioni e i cristiani non cattolici. Il suo pontificato sviluppa - seguendo il suo stile e il suo carisma - una maniera originale di mettersi in relazione con le culture e le società contemporanee. L'identità cattolica che promuove e propone non è dunque un'identità polemica o cauta, ma un'identità aperta e in dialogo. È chiaro che Giovanni Paolo II propone la "differenza cristiana" nel suo rapporto con il mondo e con gli altri, ma la singolarità cristiana non si costruisce per reazione o opposizione. Infine, possiamo talvolta osservare un certo scarto (o una certa tensione) tra i suoi gesti e i documenti pubblicati dalla Santa Sede nel corso del suo pontificato.

Se prima abbiamo parlato di una non-coincidenza tra la ricezione kerigmatica del Concilio durante il pontificato di Paolo VI e l'ethos spirituale e culturale della prima generazione chiamata a recepire il Concilio, qui possiamo parlare di una sincronia tra lo stile di Giovanni Paolo II e la nuova generazione. Un reale orizzonte di attesa corrisponde ai suoi inviti ad adottare un cattolicesimo militante, senza paura né compromessi, dai contorni netti, il cui insegnamento è chiaro e senza ambiguità e le cui pratiche sono ben definite. Una generazione che ha la sensazione di non aver avuto l'occasione di iscriversi in una tradizione, che disponesse di riferimenti chiari in merito a ciò che bisogna credere e a ciò che bisogna fare, ma di avere ricevuto in lascito solo un'appartenenza fiacca al cattolicesimo e un riferimento confuso ai suoi insegnamenti, riceveva con gioia un discorso che le offriva punti di riferimento e quadri, oltre a fornirle un'esperienza forte di appartenenza. Questi convertiti, dopo un tempo di erranza spirituale e religiosa, trovavano finalmente segnali chiari e un gruppo di appartenenza. 
È in seguito che la situazione si complica perché vari gruppi possono tentare di trarre profitto dalla situazione, volendo attrarre a sé quelle nuove aspirazioni o strumentalizzare quei convertiti per proprio interesse. Vi sono dunque vari piani da distinguere - che si incrociano, si intersecano e interagiscono tra loro - se vogliamo analizzare con un po' di precisione gli sviluppi in corso. Oltre ai livelli (interazioni tra i pastori e gli altri fedeli), ci sono in orizzontale dei piani costituiti da gruppi o da correnti che non hanno esattamente gli stessi interessi, gli stessi programmi e le stesse strategie. Capita che quelli che hanno veri e propri programmi 'politici' circa il Vaticano II strumentalizzino la nuova generazione servendosene per l'avanzamento o la realizzazione della propria causa.

\section{Un rapporto col mondo e con gli altri da costruire}

Come sottolineavamo in precedenza, la ricezione del Vaticano II da parte di questa nuova generazione si gioca in larga misura su due poste in gioco connesse: l'affermazione della differenza cristiana ovvero la costruzione dell'identità del credente in un mondo pluralista e, correlativamente, la relazione con il mondo, con la cultura e con gli altri. In una intervista concessa giovedì 17 febbraio 2010 al sito tedesco Kathnews, il superiore per la Germania della Fraternità san Pio X ha segnalato che i temi più trattati durante le discussioni in corso con la Congregazione per la dottrina della fede toccano "la questione della nuova liturgia [...], ma anche l'ecumenismo, il ruolo delle altre religioni, la relazione della Chiesa con il mondo"12". Qui si tratta non solo di temi centrali nel Vaticano II, ma di temi che si legano all'identità e all'incontro con gli altri. Insomma, le loro questioni rientrano meno nell'ecclesiologia nel senso istituzionale del termine. Rientrano nell'ecclesiologia per quanto quest'ultima riflette sui rapporti della Chiesa con il mondo, sulla cultura (iper)moderna, sui non-cristiani e sui non-cattolici e traccia confini chiari tra quelli che sono dentro e quelli che sono fuori. Rientrano nell'ecclesiologia anche per quanto la si riduce a quel che possiamo aspettarci dall'autorità della

12 Si veda "LA CROIX", 23 febbraio 2010. 
Chiesa: una parola chiara, che ripeta oggi e negli stessi termini ciò che veniva affermato ieri, che tracci chiaramente la via che dobbiamo seguire, che indichi la verità da seguire.

Per questa generazione di cristiani convertiti e in minoranza, il rapporto col mondo è da costruire e anche, di riflesso, la loro identità di cristiani. Apparentemente la questione non sembrava preoccupare la prima generazione che è stata chiamata a recepire il Vaticano II. In Occidente, i membri di quella generazione vivevano in una società dove i cattolici erano ancora in larga maggioranza ed erano cristiani per nascita, un cristianesimo ricevuto dal loro ambiente familiare e sociale. La nuova generazione, invece, oltre a non essere stata introdotta alla tradizione cristiana da un 'bagno ecclesiale', vive in un ambiente radicalmente pluralizzato sul piano delle opzioni religiose e delle convinzioni morali e filosofiche. Tutto ciò concorre a rendere fragile l'identità cattolica dei membri di questa generazione o a renderla inquieta col rischio che la ricerca di identità si muti in intransigenza e che la ricerca della verità si trasformi in difesa di posizioni rigide, il che è esattamente il contrario della ricerca. Il pluralismo esacerbato delle nostre società e la cancellazione dei riferimenti religiosi nello spazio pubblico, o addirittura la loro rimozione nel privato, può portare al riaffiorare talora violento delle identità religiose e alle regressioni integraliste. La ricerca di identità può allora essere travestita e assumere forme malsane. Il bisogno di affermarsi può condurre anch'esso al desiderio di imporre la propria verità e di farla valere in modo non solo fragoroso e inopportuno, ma talvolta anche violento e settario.

Vediamo dunque disegnarsi nettamente la differenza tra due generazioni su tali questioni cruciali. Una prima si preoccuperebbe poco della sua identità religiosa mentre, per la seconda, tale questione è capitale. In più, la prima non si sentiva minacciata dagli altri e si mostrerebbe piuttosto disposta ad apprendere da essi nel quadro di scambi e di un dialogo, mentre la seconda sarebbe più preoccupata di affermare la propria differenza e di tracciare dei confini. Infine, la prima si sentiva a suo agio nel suo mondo e ben inserita nella cultura dell'epoca, mentre la seconda vivrebbe un rapporto conflittuale con la società e la cultura che non le hanno trasmesse la fede. A prima vista dunque la ricezione del Vaticano II sarebbe ostacolata dal ben noto caso del conflitto generazionale. Il Vaticano II 
parlerebbe alla generazione dei baby-boomers, aperti ad accomodamenti con la cultura della propria epoca e desiderosi di adattarsi al mondo, mentre non avrebbe più niente da dire ad una generazione di convertiti, più inclini a rimarcare la propria differenza e ad affermare la propria singolarità. Il cambiamento di contesto renderebbe dunque caduche le opzioni del Concilio sulla questione capitale del rapporto con il mondo, con le culture e con gli altri. Concilio pastorale, il suo insegnamento varrebbe soltanto in un contesto dato, come lo si intende talvolta, sebbene, essendo cambiata la situazione, le sue opzioni siano di scarso aiuto oggi per la nuova generazione.

Stando così le cose, sarebbe vano interessarsi alla ricezione del Vaticano II da parte della nuova generazione. Il Concilio non soltanto è un evento che appartiene definitivamente al passato e non fa parte del campo d'esperienza e di riferimento di questa generazione, ma non è per essa di alcuna pertinenza poiché sarebbe responsabile della crisi della Chiesa e della situazione nella quale si trovano la società e la cultura occidentali. Le sue proposizioni e il suo insegnamento sono da oltrepassare, se non da rivedere, in un contesto che è radicalmente cambiato. È del resto su questa posizione che vive il movimento dell'ortodossia radicale. Se non si coltiva più il sogno di rifare una società cristiana - una città cattolica -, si spera almeno di potere contestare il mondo presente collocandosi in una marginalità contestatrice, sia della Chiesa post-conciliare sia della cultura attuale, raggruppandosi in nicchie, isolotti cattolici in un mondo ostile. In queste nicchie protettrici, si vuole restaurare il cattolicesimo di sempre il che rende chiara l'identità - e si intrattengono rapporti polemici con la cultura e relazioni distanti con gli altri, incoraggiando un rigido regime di separazione che protegge da ogni contaminazione.

Non posso comunque pensare che questa sia la sola ipotesi possibile. In effetti, malgrado le differenze che ho voluto sottolineare tra questa generazione e il concilio Vaticano II, vi è indubbiamente un orizzonte comune e non semplicemente un'opposizione irriducibile. L'orizzonte comune deriva dal fatto che la questione centrale per questa generazione, quella del rapporto con la cultura, con il mondo e con gli altri (non-cristiani, cristiani non-cattolici, ecc.), è stata una delle questioni cruciali per i Padri del concilio e al centro dell'insegnamento del Vaticano II. Insomma, entrambe 
pongono la stessa questione. Non è dunque impossibile allora instaurare una discussione prendendo come punto di partenza tale problematica comune, questione che si pone naturalmente nei due diversi contesti culturali e alla quale i due gruppi sembrano dare una risposta radicalmente diversa.

La ricezione, ci insegnano gli specialisti, "risulta dalla fusione di due orizzonti: quello dell'autore che le ha dato forma e senso, quello del pubblico che interpreta e reinterpreta continuamente questa forma e questo senso in funzione dell'attualità" (JAUSS, 1978, p. 90). Siamo dunque in presenza, sulla questione del rapporto con il mondo e con gli altri, di due orizzonti: quello dei Padri conciliari che hanno proposto un insegnamento conciliare e quello del nuovo pubblico costituito dalla generazione di giovani cattolici spesso convertiti. Si tratterebbe dunque oggi di intraprendere una rilettura dei testi conciliari che permettesse la fusione di quei due orizzonti, o meglio di favorire un dialogo, o una messa in discussione dei presupposti sui quali si basano i due orizzonti. Questo ci condurrà a comprendere in altro modo la storicità dell'insegnamento conciliare che non sarà acquisito soltanto ricollocandolo nel suo contesto di produzione e ricostruendo la storia della sua redazione, ma instaurando un dialogo fra l'autore (il Concilio), l'opera (il testo) e un nuovo pubblico-lettore ${ }^{13}$.

Ciò ci pone oggi di fronte a una nuova sfida in questa tappa della ricezione del Vaticano II. In effetti, non si tratta più oggi di proseguire senza fine il lavoro colossale compiuto nel corso degli anni post-conciliari e che ha condotto a ricostruire la storia dei testi e dell'evento conciliare credendo che, così facendo, saremmo condotti a conoscere il senso di quei testi, senso che si tratterebbe, a cose fatte, solo di trasmettere. Per riprendere la critica che Jauss rivolge alla storia letteraria, si è forse creduto che si trattasse solo di

spiegare l'opera d'arte [il concilio nel nostro caso] attraverso la somma delle sue determinazioni storiche [e] per ogni opera riprendere integralmente

13 Jauss denuncia il paradigma positivista talora adottato in storia, che ha "ridotto la complessità dell'esperienza letteraria alla semplicità di un concatenamento causale fra le opere e gli autori, la comunicazione specifica della storicità letteraria che si stabilisce fra l'autore, l'opera e il pubblico è scomparsa dietro una successione di monografie, ipostatizzate in una 'storia' di cui non aveva più di fatto che il nome”. 
la ricerca, al fine di raggiungere attraverso le fonti le 'origini', e attraverso la vita dell'autore le determinazioni che sono di pertinenza dell'epoca e dell'ambiente. Cercando le fonti, si è inevitabilmente condotti a cercare anche le fonti delle fonti e a perdersi nelle 'storie' (JAUSS, 1978, p. 82).

Ci si impegna allora in una storia senza fine, fino alla nausea, senza tuttavia che una discussione fruttuosa possa stabilirsi tra l'opera e il pubblico lettore. È senza dubbio di questa discussione che abbiamo bisogno oggi, se speriamo di fare un passo in più nella ricezione del Concilio. È senza dubbio questa la sfida che ci attende oggi.

\section{Conclusioni}

Ho voluto, limitandomi all'Occidente o allo spazio Nord-Atlantico (benché bisognerebbe fare ancora importanti sfumature), mostrare fino a che punto il processo di ricezione sia complesso e operi a molteplici livelli e su differenti piani. Non basta, per esempio, interpretare la ricezione a partire da un pontificato o dalle iniziative degli organismi centrali di governo della Chiesa cattolica. Bisogna vedere in che misura queste iniziative trovino eco presso una generazione. Bisogna anche esaminare le interazioni tra i diversi gruppi nella Chiesa. Insomma, bisogna esaminare i rapporti e le relazioni piuttosto che le semplici azioni o, se bisogna tener conto delle azioni, bisogna situarle in un sistema dinamico. Questo è richiesto anche da una opzione ecclesiologica di base: la ricezione riguarda il pleroma della Chiesa e non semplicemente un attore.

Ho voluto anche mettere in rilievo l'importanza del fattore generazionale nella ricezione del Vaticano II piuttosto che insistere semplicemente sul contesto della ricezione che si sarebbe modificato. A mio parere, gli studi hanno insistito troppo finora sulla modificazione del contesto (ACERBI, 1981; VAUCELLES, 1985). e non abbastanza sui mutamenti generazionali prodotti dai mutamenti dei contesti ma che non si confondono con essi. 


\section{Riferimenti}

ACERBI, A. La recezione del Concilio Vaticano II in un contesto storico mutato. Concilium, v. 6, n. 166, p. 137-149, 1981.

ATTIAS-DONFUT, C. Générations et âges de la vie. Paris: Presses Universitaires de France, 1991.

AUTRICE. Sociologie des générations: l'empreinte du temps. Paris: Presses Universitaires de France, 1988.

BUONASORTE, N. Tra Roma e Lefebvre: Il tradizionalismo cattolico italiano e il Concilio Vaticano II. Roma: Studium, 2003.

CHAMPIGNY, P.; ETEVE, C. Dictionnaire de l'éducation et de la formation. Paris: Nathan, 1994.

GALLAND, O. Sociologie de la Jeunesse. Paris: A. Colin, 1997.

DELUMEAU, J. Déchristianisation ou nouveau modèle de christianisme? Archives des Sciences Sociales des Religions, v. 40, n. 40, p. 3-20, 1975.

DUMONT, F. Âge, générations, société de la jeunesse. In: DUMONT, F. Une société des jeunes. Québec: l'IQRC, 1986. p. 15-28.

GALLAND, O. Sociologie de la jeunesse: l'entrée dans la vie. Paris: A. Colin, 1991. Réédité en 1997 et en 2001.

GRAND’MAISON, J. Le défi des générations: enjeux sociaux et religieux du Québec d'aujourd'hui. Montréal: Fides, 1995.

HOWE, N.; STRAUSS, W. The history of America's future, 1584 to 2069. New York: Quill, 1991.

HOWE, N.; STRAUSS, W. 13th gen: abort, retry, ignore, fail? New York: Vintage Books, 1993.

HOWE, N.; STRAUSS, W. The fourth turning: an American prophecy. New York: Broadway Books, 1997. 
HOWE, N.; STRAUSS, W. Millennials rising: the next great generation. New York: Vintage Books, 2000.

HOWE, N.; STRAUSS, W. Millennials go to college: strategies for a new generation on campus. Washington: American Association of Collegiate Registrars, 2003.

HOWE, N.; STRAUSS, W. Millennials and the pop culture: strategies for a new generation of consumers. Great Falls: Lifecourse Associates, 2006a.

HOWE, N.; STRAUSS, W. Millennials go to college surveys and analysis: from boomer to gen-x parents. Great Falls: Lifecourse Associates, 2006b.

JAUSS, H. R. Pour une esthétique de la réception. Paris: Gallimard, 1978.

KESSLER, D.; MASSON, A. Cycles de vie et générations. Paris: Économica, 1985.

LOTTIN, A. Contre-Réforme et religion populaire: un mariage difficile et réussi aux XVI ${ }^{e}$ et XVII ${ }^{e}$ siècles en flandre et en ainaut? In: DUBOSCQ, G.; PLONGERON, B.; ROBERT, D. Religion populaire. Paris: Editions du Centre National de la Recherche Scientifique, 1979. p. 53-63.

MANNHEIM, P. K. Si veda il suo classico le generazioni. Bologna: Il Mulino, 2008.

MATTE, I. Rite de passage en contexte moderne: l'exemple de la scène musicale underground montréalaise. 2000. 130 f. Tesi (di Laurea Anthropologie) Dipartimento di antropologia, Università Laval, 2000.

MATTE, I. Malaise existentiel et discours apocalyptique dans la jeune chanson québécoise. In: MAGER, R.; CANTIN, S. Modernité et religion au Québec: où en sommes-nous? Québec: PUL, 2009. p. 165-179.

O'MALLEY, J. The resiliency of the institution and its style, in four cultures of the west. London: Harvard University Press, 2004.

O’MALLEY, J. Quattro culture dell'Occidente. Milano: Vita e Pensiero, 2007. ORTEGA Y GASSET, J. Tema del nostro tempo. Milano: Rosa e Ballo, 1994. 
PELLETIER, D. La crise catholique. Paris: Payot, 2002.

POTTMEYER, H. J. Vers une nouvelle phase de réception de Vatican II. Vingt ans d'herméneutique du Concile. In: ALBERIGO, G.; JOSSUA J.-P. La réception de Vatican II. Paris: Éd. du Cerf, 1985. p. 43-64.

ROUTHIER, G. La périodisation. In: ROUTHIER, G. Réception du Vatican II. Le Concile au risque de l'histoire et des espaces humains. Leuven: Peeters, 2004. p. 225-244.

ROUTHIER, G. Le Concile Vaticane II livré aux interprétations de générations successives. Science et Esprit, v. 91, n. 2/3, p. 237-255, 2009.

ROUTHIER, G. La ricezione del Concilio. Mentalità, soggetti e tempo di un percorso laborioso. Rivista del Clero Italiano, v. 91, n. 4, p. 263-285, 2010.

VAUCELLES, L. de. Les mutations de l'environnement sociétaire du catholicisme durant la période post-conciliaire. In: ALBERIGO, G.; JOSSUA, J.-P. La réception du Vatican II. Paris: Éd. du Cerf, 1985. p. 65-84.

Ricevuto: 12/12/2011

Recebido: 12/12/2011

Approvato: 20/02/2012

Aprovado: 20/02/2012 